\title{
EL POSICIONAMIENTO DE LA TEORÍA DE LA AUTORÍA MEDIATA POR ORGANIZACIÓN EN LA JURISPRUDENCIA PERUANA* \\ Análisis de la fundamentación dogmática de la Sentencia de la Corte Suprema contra Alberto Fujimori
}

\author{
RAÚl PARIONA ARANA**
}

\begin{abstract}
Resumen
En histórica sentencia, sin precedentes en la jurisprudencia nacional, y una de las más relevantes de la jurisprudencia internacional, la Sala Penal Nacional de la Corte Suprema de la República condenó al ex presidente Alberto Fujimori Fujimori como autor mediato de crímenes contra la humanidad, basándose escencialmente dicho fallo en la teoría de la autoría mediata por organización para el enjuiciamiento de los crímenes cometidos desde aparatos organizados de poder. El estudio realiza un recuento del origen y los antecedentes de la teoría. Se analiza y formulan apreciaciones críticas a la amplia fundamentación de la sentencia respecto a la participación criminal que desplegó el ex mandatario peruano, la misma que sienta ya las bases de una doctrina jurisprudencial nacional y consolida la autoría mediata en nuestro medio.
\end{abstract}

Palabras clave: Autoría mediata - Aparatos de poder organizados - Dominio sobre la organización - Poder de mando - Fungibilidad - Elevada disposición al hecho del ejecutor - Crímenes contra la humanidad.

\begin{abstract}
In a historical ruling, unprecedented in national law, and one of the most relevant sentences in international case law, the National Criminal Division of the Supreme Court of Peru convicted the former President Alberto Fujimori Fujimori as the indirect perpetrator of crimes against humanity, essentially on the grounds of the theory of the indirect perpetrator by organization, to judge those crimes perpetrated through organized power structures. The study recalls the origin and background of the theory, analyzing and providing critical opinions on the grounds of the sentence in relation to the criminal participation of the former Peruvian head of state, which sets the bases for national case law and consolidates the indirect perpetratorship in our midst.
\end{abstract}

\footnotetext{
* El presente trabajo es la materialización en papel de la conferencia dictada en la Unidad de Postgrado de la Universidad San Marín de Porres en el marco del Simposium sobre la Sentencia en el caso Fujimori que tuvo lugar el día 17 de abril de 2009. Expreso aquí mi más grande agradecimiento a los profesores Felipe Villavicencio Terreros y Edwar Álvarez Yrala por haberme brindado la oportunidad de exponer mis ideas en tan prestigioso claustro.

** Profesor de la Universidad Nacional Mayor de San Marcos, Pontificia Universidad Católica del Perú y Universidad San Martín de Porres.
} 
Raúl Pariona Arana - El posicionamiento de la teoría de la autoría mediata por organización en la jurisprudencia peruana

Key words: Indirect Perpetratorship - Organized Power Structures - Power over the organization - Commanding Power - Fungibility - Willingness to comply with the wishes of the executor - Crimes against humanity.

\section{Sumario}

1. Consideraciones previas. 2. La teoría de la autoría mediata por organización en la ciencia y en la jurisprudencia - antecedentes. 3. Consolidación de la autoría mediata por organización en la doctrina y jurisprudencia. 4. La autoría mediata por organización según la Corte Suprema del Perú en la sentencia contra Alberto Fujimori. 5. Consideraciones sobre la concepción asumida en la sentencia. 6. Propuesta de fundamentación de la autoría mediata por organización - Concepción personal. 7. Palabras finales.

\section{CONSIDERACIONES PREVIAS}

El 7 de abril de 2009 la Sala Penal Especial de la Corte Suprema del Perú condenó al ex presidente Alberto Fujimori Fujimori como autor mediato de crímenes contra la humanidad por el asesinato y posterior desaparición de un profesor y nueve estudiantes de la Universidad Enrique Guzmán y Valle (La Cantuta), por la muerte de diecisiete personas en una quinta del distrito limeño de Barrios Altos, y por el secuestro de un empresario y un periodista.

Se trata de una sentencia sin precedentes en la historia denuestrajurisprudencia y, con seguridad, de una de las más relevantes de la jurisprudencia internacional. Para fundamentar el tipo de participación criminal que desplegó el ex mandatario peruano, la sentencia se basó en la teoría de la autoría mediata por organización. En la sentencia se puede apreciar además, una fundamentación amplia y profunda de este tipo de autoría, que sienta ya la bases de una doctrina jurisprudencial nacional. Dado el desarrollo alcanzado por la teoría de la autoría mediata por organización para el enjuiciamiento de los crímenes cometidos desde aparatos organizados de poder, la asunción de esta figura dogmática por parte de la Corte Suprema debe ser valorada positivamente.

En las páginas que siguen se realiza un análisis de la fundamentación de la autoría mediata por organización que la Corte Suprema ha realizado; en esta línea, se realiza un recuento del origen y los antecedentes de la teoría, se analiza y valora la concepción de la Corte Suprema expuesta en la sentencia, y finalmente, se presenta la concepción que proponemos para fundamentar la autoría mediata por organización. 
Raúl Pariona Arana - El posicionamiento de la teoría de la autoría mediata por organización en la jurisprudencia peruana

\section{LA TEORÍA DE LA AUTORÍA MEDIATA POR ORGANIZACIÓN EN LA CIENCIA Y EN LA JURISPRUDENCIA - ANTECEDENTES}

La teoría de la autoría mediata por dominio de organización fue fundada por el profesor alemán Claus Roxin. En su conferencia ${ }^{1}$ pronunciada con motivo del inicio de clases en Hamburgo propuso, por vez primera en la ciencia del Derecho penal, un nuevo supuesto de autoría mediata. Sobre la base del criterio del dominio del hecho elaboró una tesis que permitiría imputar responsabilidad penal a título de autores a aquellos que sin ejecutar los hechos directamente se limitaban a dar las órdenes para su comisión. La teoría de Roxin implicó en la doctrina la renovación del entendimiento de la autoría mediata, pues hasta entonces, en principio solo se podía imputar responsabilidad a título de autores a quienes sin realizar directamente los hechos, se valían de personas que actuaban sin dolo o actuaban bajo amenaza. El problema que hasta entonces se presentaba era determinar cómo se podía hacer responsable al hombre de atrás por hechos que había realizado otra persona plenamente responsable. Roxin ${ }^{2}$ planteó la siguiente solución: el "hombre de atrás" domina la voluntad del ejecutante si da una orden de ejecución del hecho punible a través de un aparato de poder. El autor no necesita aquí coaccionar o engañar al ejecutor, incluso ni siquiera conocerlo, pues el aparato de poder organizado garantiza por sí solo la ejecución del hecho punible, ya que en caso de incumplimiento de la orden por parte de un determinado ejecutante, hay siempre otro que lo reemplazará en la ejecución ${ }^{3}$. La constelación de casos que Roxin tuvo presente en la elaboración de su teoría fueron los crímenes ordenados por Hitler, Himmler, Eichmann y otros mandos políticos y militares del gobierno nacional socialista. Para él, en tales casos quienes dictaron las órdenes de exterminio eran autores mediatos, pues ellos estaban colocados en la palanca de un aparato de poder y a través de las órdenes impartidas dieron lugar a delitos en los cuales resultó irrelevante la individualidad de los ejecutantes ${ }^{4}$.

Roxin desarrollo y amplió su teoría en posteriores trabajos ${ }^{5}$. En la actualidad la estructura dogmática de su teoría presenta características bien definidas.

\footnotetext{
1 Su conferencia fue publicada ese mismo año en la revista GA 1963, p. 193 y ss., bajo el título "Delitos en el marco de aparatos de poder organizado". Esta exposición pasó a formar el § 24 de su libro "Täterschaft und Tatherrschaft" publicado en ese mismo año.

2 RoxIN, GA 1963, p. 193, 200; el mismo, Täterschaft und Tatherrschaft, 2006, pp. 242, 243; el mismo, AT II, § 25, n. marg. 45, 46, 105.

3 ROXIN, AT II, § 25, n. marg. 107.

4 Roxin, en: Problemas actuales de la dogmática penal, traducción de Manuel A. Abanto Vásquez, 2004, p. 224. (cit.: Problemas actuales)

5 Cfr. FS-Schroeder, 2006, pp. 387 y ss.
} 
Raúl Pariona Arana - El posicionamiento de la teoría de la autoría mediata por organización en la jurisprudencia peruana

Según su opinión, para afirmar una autoría mediata en los casos objeto de estudio debe presentarse, en primer lugar, un "poder de mando sobre la organización". Este dominio supone en el agente la capacidad de dar órdenes que deben cumplirse para el funcionamiento mismo de la organización, siendo indiferente el nivel jerárquico que ocupa el agente, ya que únicamente importa que domine la parte de organización a él sometida. En segundo lugar, la organización de la cual se sirve el hombre de atrás debe encontrarse "al margen del Derecho". Pertenecen a este tipo de organización los aparatos de poder estatales, organizaciones criminales comunes, como también organizaciones terroristas. En tercer lugar, en la organización en concreto debe existir la posibilidad de reemplazar al actor inmediato, es decir, debe concurrir la fungibilidad del ejecutor. El actor inmediato sería solamente una figura intercambiable, un "engranaje" reemplazable en la maquinaria del aparato de poder" 6 . Finalmente, como cuarto elemento que ha sido desarrollado en sus últimos trabajos sobre el tema, Roxin considera que debe concurrir una alta disposición al hecho del ejecutor ${ }^{7}$.

\section{CONSOLIDACIÓN DE LA AUTORÍA MEDIATA POR ORGANIZACIÓN EN LA DOCTRINA Y JURISPRUDENCIA}

En la actualidad, la teoría de la autoría mediata en virtud de dominio por organización se ha impuesto y constituye opinión dominante ${ }^{8}$ no solo en Alemania, sino también en gran parte de los países de tradición romanogemánica como el nuestro. En el Derecho penal internacional ${ }^{9}$ tampoco ha pasado desapercibida. Su influencia en este ámbito ha sido tal que, conforme lo pone de relieve Satzger ${ }^{10}$, habría sido asumida expresamente en el Estatuto de la Corte Penal Internacional. La fuerza convincente de esta teoría también ha motivado su aceptación por parte de la jurisprudencia. En Alemania ésta ha sido asumida por la Corte Suprema en su famosa sentencia ${ }^{11}$ contra los miembros del Consejo de Defensa Nacional de la ex República Democrática

\footnotetext{
6 Cfr. RoxIN, Täterschaft, pp. 224, 225.

7 RoxIN, FS-Schroeder, 2006, pp. 387 y ss.

8 Aquí son mencionados por su importancia los trabajos de MEINI. El dominio de la organización en el derecho penal. Lima, 2008; MONTOYA VIVANCO. En: Problemas fundamentales de la parte general del código penal, Lima, Friburgo 2009, p. 61 y ss.; CASTILlo Alva. En: Libro Homenaje a Enrique Bacigalupo, pp. 576 y ss. Para una detallada referencia de la doctrina que sigue esta teoría véase mis trabajos en: La autoría mediata por organización. Lima, 2009.

9 AMBOS. Internationales Strafrecht. 2005, §7, n. marg. 27 y s.; el mismo, Der Allgemeine Teil des Völkerstrafrechts. Ansätze einer Dogmatisierung, 2002, p. 568; Kreß, GA 2006, p. 307; RADKE, GA 2006, p. 350. SATZGER, Internationales Strafrecht, § 14, n. marg. 43; Vest, ZStW 113 (2001), p. 457; Vogel, ZStW 114 (2002), S. 427; Werle, Völkerstrafrecht, 2003, n. marg. 407.

10 SATZGER, Internationales Strafrecht, § 14, n. marg. 43.

11 BGHSt. 40, 218 que se remonta al 26 de julio de 1994.
} 
Raúl Pariona Arana - El posicionamiento de la teoría de la autoría mediata por organización en la jurisprudencia peruana

de Alemania el caso de los disparos del muro y, desde aquel entonces, en otras sentencias de gran trascendencia.

En nuestra región son importantes las resoluciones emitidas por los tribunales argentinos que juzgaron a la cúpula militar que gobernó ese país. Así, también lo es la sentencia de extradición en el caso Fujimori emitida por la Corte Suprema de Chile que asume expresamente la autoría mediata por organización. En nuestro país tenemos como antecedentes varias sentencias emitidas por Salas Superiores de Justicia y aquellas emitidas por la Corte Suprema; aquí resulta relevante la sentencia emitida en el caso Abimael Guzmán.

Ahora, con la sentencia contra Fujimori, la cual es objeto de comentario, se consolida en la ciencia y la jurisprudencia nacional e internacional la teoría de la autoría mediata por organización como la más adecuada estructura dogmática para juzgar crímenes cometidos desde y mediante aparatos organizados de poder. Ésta es el resultado de una evolución que debe ser calificada positivamente. La sentencia deja ver un alto nivel jurídico-científico de nuestra Corte Suprema, que con seguridad convertirá a nuestra jurisprudencia en un referente elevado.

\section{LA AUTORÍA MEDIATA POR ORGANIZACIÓN SEGÚN LA CORTE SUPREMA DEL PERÚ EN LA SENTENCIA CONTRA ALBERTO FUJIMORI}

La fundamentación de la autoría mediata por organización realizada por la Corte Suprema es expuesta en el parágrafo tercero del capítulo segundo de la tercera parte de la sentencia referida a los fundamentos jurídicos penales ${ }^{12}$. En el indicado parágrafo titulado "la autoría mediata por dominio de la voluntad en aparatos de poder organizados" la sentencia - en clara asunción de las tesis del profesor Claus Roxin - presenta la siguiente fundamentación: la autoría mediata por organización tendría como soporte fundamental la "existencia previa de una organización estructurada", vertical y jerarquizada. Ésta debe poseer "una línea jerárquica sólida que hará responsable a su nivel estratégico superior por las decisiones y designios de carácter delictivo que a su interior se adopten. Los cuales, luego, le serán asignados al ejecutor inmediato por la vía de la verticalidad que presenta su diseño organizacional"13.

Según la Corte, dos serían las características de esta organización. La primera característica sería la "asignación de roles" ${ }^{14}$ que designaría la "relación entre

\footnotetext{
12 Ver Sentencia, pp. 629-657.

13 Sentencia, p. 633.

14 Ibid.
} 
Raúl Pariona Arana - El posicionamiento de la teoría de la autoría mediata por organización en la jurisprudencia peruana

el nivel estratégico y el ejecutor, y que aluden a una división del trabajo o distribución de funciones". La segunda característica sería el "desarrollo de una vida funcional que es independiente a la de sus integrantes"; 15 cuyo fundamento "no radica en un estado de ánimo especial del nivel superior estratégico, sino en el "mecanismo funcional del aparato", esto es, su "automatismo" o desarrollo de un proceso o funcionamiento por sí sólo". Producto de esto, "el hombre de atrás podrá confiar siempre en que su orden o designio criminal se van a cumplir sin necesidad de que tenga que conocer al ejecutor inmediato", este "funcionamiento automático del aparato" sería aquello que "realmente garantice el cumplimiento de la orden"16.

Para la sentencia, la autoría mediata por organización requiere de la presencia de "presupuestos y requisitos funcionales". Estos serían i) el poder de mando; ii) la desvinculación de la organización del ordenamiento jurídico; iii) la fungibilidad del ejecutor inmediato; y iv) la elevada disposición del ejecutor hacia el hecho. La Corte clasifica a su vez estos requisitos en dos niveles, un nivel objetivo y un nivel subjetivo. El primero comprendería al "poder de mando" y "la desvinculación del ordenamiento jurídico del aparato de poder". El poder de mando "resulta trascendental para materializar el dominio de la organización", mientras que la desvinculación del derecho "le dará mayor solidez a este dominio". Basada en estas características, la sentencia califica a ambos como "el soporte básico que permitirá al nivel estratégico superior (autor mediato) edificar y consolidar su dominio sobre la totalidad de la estructura criminal."17 Por otra parte, el nivel subjetivo comprende a la "la fungibilidad del ejecutor directo" y su "elevada disponibilidad hacia la realización del hecho". Estos elementos permitirían inferir "que la actuación del ejecutor directo dependerá finalmente de su propia voluntad a la realización del hecho. En cambio, la no ejecución por éste del evento criminal, conllevará a su fungibilidad o sustitución por otra persona interpuesta que tenga una mayor predisposición a la realización del hecho típico"18.

\section{CONSIDERACIONES SOBRE LA CONCEPCIÓN ASUMIDA EN LA SENTENCIA}

La asunción de la autoría mediata por organización hecha por la CorteSuprema es un paso importante en el desarrollo de nuestra doctrina jurisprudencial y consolida esta forma de autoría en nuestro medio. Pese a este importante paso

\footnotetext{
15 Ibid.

16 Ibid.

17 Sentencia, p. 634

18 Sentencia, p. 635.
} 
Raúl Pariona Arana - El posicionamiento de la teoría de la autoría mediata por organización en la jurisprudencia peruana

en el desarrollo de la doctrina jurisprudencial sobre esta figura dogmática, la fundamentación realizada no está exenta de algunas apreciaciones críticas.

Sobre la admisión del "Poder de mando" y el "apartamiento del derecho" como presupuestos de la autoría mediata por organización -que la sentencia califica de "soporte básico"19- no queda sino saludar la sentencia. Ésta constituye pues el camino correcto en la fundamentación. Es más, debe subrayarse que justamente en el desarrollo y fundamentación de estos dos presupuestos radica la contribución más importante de la sentencia. Allí se indica, de modo correcto, siguiendo la doctrina más moderna, que el dominio que el hombre de atrás ejerce en la materialización del resultado criminal radica en el poder de mando que tiene sobre la organización la que se encuentra al margen del derecho.

La sentencia señala que el primer elemento ("objetivo") "poder de mando" es condición fundamental, para imputar autoría mediata en el marco de un aparato de poder organizado ${ }^{20}$. Esto es correcto. La sentencia ${ }^{21}$ profundiza y afirma que el poder de mando es "la capacidad del nivel estratégico superior -del hombre de atrás- de impartir órdenes o asignar roles a aquella parte de la organización que le está subordinada. Esta capacidad la adquiere, o le puede ser conferida, en atención a una posición de autoridad, liderazgo o ascendencia derivadas de factores políticos, ideológicos, sociales, religiosos, culturales, económicos o de índole similar. El poder de mando del autor mediato se manifiesta ejercitando órdenes, de modo expreso o implícito, las cuales serán cumplidas debido a la automaticidad que otorga la propia constitución funcional del aparato, es decir, sin que sea necesario que quien ordena deba además, o alternativamente, recurrir a la coacción o al engaño de los potenciales ejecutores".

Se afirma correctamente que el poder de mando constituye un elemento objetivo. Esto es evidente y no precisa mayor argumentación. La asunción del elemento "poder de mando" como "presupuesto" del dominio por organización es también correcta, pues el poder de mando es el elemento fundamental que fundamenta la autoría mediata por organización. Con este elemento se revela pues lo que en trabajos anteriores he denominado el dominio real y concreto sobre la organización. El poder de mando es sin duda el elemento que expresa de manera fáctica este dominio.

\footnotetext{
${ }^{19}$ Sentencia, p. 634.

${ }^{20}$ Sentencia, p. 635.

${ }^{21}$ Ibid.
} 
Raúl Pariona Arana - El posicionamiento de la teoría de la autoría mediata por organización en la jurisprudencia peruana

El segundo elemento ("objetivo") fundamentador de la autoría mediata por organización mencionado por la sentencia es el "apartamiento del Derecho". $\mathrm{Al}$ respecto, acierta la sentencia cuando afirma que este elemento se trata de un "presupuesto". Igualmente, la inclusión de este elemento en un nivel objetivo no merece objeción alguna. Se debe resaltar como positivo el desarrollo que sobre este elemento realiza la sentencia. La sentencia indica que "la admisión de la autoría mediata del nivel estratégico superior quedará condicionada a que las órdenes de este estamento sean dictadas en el marco de una organización que opera al margen del ordenamiento jurídico del "Estado de Derecho"22. Luego refiere, "la desvinculación del ordenamiento jurídico en la criminalidad estatal puede darse de dos maneras. Primero, cuando el nivel superior estratégico del Estado decide apartarse por completo del Derecho y crear un sistema normativo totalmente diferente que no es reconocido ni aceptado por el Derecho internacional, en tanto expresa o encubre la comisión de delitos graves. Segundo, cuando el nivel superior estratégico del poder estatal se aleja paulatinamente del ordenamiento jurídico; inicialmente solo para la realización de determinados hechos punibles, pero luego, con actos sistemáticos cada vez más frecuentes, así como a través de acciones tendientes a anular, desnaturalizar o sustituir distorsionadamente los diferentes ámbitos y competencias que configuran los estamentos oficiales, legales y de control del Estado. Esta modalidad resulta ser la más grave porque se cubre de una aparente legitimidad; sin embargo, subrepticiamente intenta crear un sistema normativo alterno al legalmente vigente, aprovechando, justamente, sus formas y estructuras para la comisión de delitos graves." ${ }^{23}$ La sentencia indica pues que el caso evaluado (el de Alberto Fujimori) se trataría de un gobierno de facto por razón de su ejercicio, es decir, de "aquellos que fueron instituidos con las formalidades estipuladas en la Constitución, pero que luego se van expresando, manifestando y conduciendo fuera de ella o contra lo previsto por ella"24.

En este marco conceptual, se identifica a "Derecho" como "un sistema u ordenamiento jurídico representado por un conjunto coordinado de normas generales y positivas que regulan la vida social. El Estado, como comunidad, define un orden normativo. Este orden normativo solo puede ser un orden jurídico, aquél que comúnmente se relaciona como el "Derecho del Estado" o el "Derecho nacional". Sin embargo, este Derecho nacional se encuentra estrechamente vinculado e integrado con el Derecho internacional constituyendo una

\footnotetext{
22 Sentencia, p. 642

23 Ibid.

${ }^{24}$ Sentencia, p. 643.
} 
Raúl Pariona Arana - El posicionamiento de la teoría de la autoría mediata por organización en la jurisprudencia peruana

unidad. Por tanto, el Derecho internacional forma parte del orden jurídico nacional en tanto que las normas producidas en el contexto internacional se incorporan al Derecho del Estado nacional. En consecuencia, el apartamiento o desvinculación del Derecho significa que la organización se estructura, opera y permanece al margen del sistema jurídico nacional e internacional" 25 . Apoyándose en Roxin, la sentencia ${ }^{26}$ indica que el apartamiento del Derecho se presenta "no sólo en delitos cometidos por órganos del Estado o aparatos del poder estatal, sino también sería aplicable a los casos de "criminalidad organizada no estatal" y en muchas "formas de aparición del terrorismo". Únicamente se debería excluir a los casos de criminalidad empresarial".

De otro lado, en la fundamentación que la Corte Suprema hace de la autoría mediata por organización aplicada en su sentencia, se afirma la existencia de un "nivel subjetivo" el cual incluye a la fungibilidad y a la elevada disposición al hecho del ejecutor. Según la sentencia, la fungibilidad constituye un presupuesto de carácter subjetivo que sirve a la imputación de una autoría mediata por dominio de la voluntad en aparatos de poder organizados ${ }^{27}$. Se menciona que la fungibilidad ha sido "entendida, generalmente, como la característica del ejecutor de poder ser intercambiado o sustituido por el nivel estratégico superior en la operativización y realización de su designio delictuoso" 28 .

Lo que inmediatamente salta a la vista es que el elemento "fungibilidad" no constituye en realidad un elemento subjetivo, sino por el contrario de un presupuesto objetivo. La fungibilidad no es un elemento interno, un estado de la mente humana o de la psiquis del hombre, como podrían ser el dolo o algunas formas de tendencias internas criminales ("animus"). La fungibilidad es reemplazabilidad, posibilidad de cambio de ejecutores. Según la teoría, fungibles serán los ejecutores directos del crimen, pues en caso no cumplan las órdenes criminales existirán otros ejecutores que los reemplazarían en la ejecución. Esta reemplazabilidad tiene carácter objetivo, pues en el caso concreto los ejecutores existen o no existen; esto no depende de la mente humana.

Otro aspecto que debe ser apreciado críticamente es que la sentencia admite erróneamente a la fungibilidad como "presupuesto" de la autoría mediata por organización, es decir, le asigna una función fundante de la autoría mediata, lo que implicaría, a su vez, que su ausencia provocará que no se configure

\footnotetext{
${ }^{25}$ Sentencia, p. 640.

26 Sentencia, p. 641.

27 Sentencia, p. 644.

28 Ibid.
} 
Raúl Pariona Arana - El posicionamiento de la teoría de la autoría mediata por organización en la jurisprudencia peruana

esta forma de autoría, es decir, si en el caso concreto se comprueba que los ejecutores no eran remplazables, entonces no se configurará una autoría mediata. Con todo, esta postura de la sentencia debe ser criticada, pues la jurisprudencia internacional nos muestra que existen constelaciones de crímenes mediante aparatos de poder donde no hubo fungibilidad, como los casos de organizaciones criminales donde los ejecutores son sujetos con conocimientos especiales que no son susceptibles de ser reemplazados fácilmente. Sin embargo, como ya lo indiqué en anteriores trabajos, esta característica no debe llevar a negar la autoría de quienes dan las órdenes desde las altas esferas de poder. La fungibilidad es una característica de la autoría mediata por organización pero no es un presupuesto de ella, es decir, no es un elemento fundante. ¡La fungibilidad acompaña - por lo general - a esta forma de autoría pero no la fundamenta!

La sentencia menciona finalmente a la "elevada disposición al hecho" como el cuarto presupuesto de la autoría mediata por organización. Este elemento tendría igualmente, carácter subjetivo. Según la sentencia, el hombre de atrás tendría el dominio del evento criminal, es decir, que estaría seguro que sus órdenes se van a cumplir porque los ejecutores directos de los crímenes serían personas que están predispuestas a cometer el hecho criminal. Es decir, los ejecutores directos serían personas con una cierta tendencia a la comisión de crímenes.

Se puede observar, al igual que en el caso de la fungibilidad, que el Tribunal atribuye a este elemento el carácter de presupuesto de la autoría mediata por organización. La asignación de este carácter significa que la ausencia de este elemento en el caso concreto llevaría a negar la autoría mediata por organización. Esta postura también debe ser criticada. Existen pues muchos casos donde esta "disposición al hecho criminal" del ejecutor no se presenta y pese a ello se puede observar un dominio real del hombre de atrás. Piénsese únicamente en los casos de los crímenes del nacional-socialismo en Alemania, donde muchos de los soldados alemanes no estaban de acuerdo con las políticas criminales implementatadas por el Estado totalitario, que no tenían "una elevada disposición al hecho criminal" de asesinar seres humanos en masa ; y que sin embargo cumplían las órdenes criminales, para no ser objeto de represalias, o para seguir ascendiendo en su carrera política o militar, o simplemente para congraciarse con "los de arriba".

El dominio del evento criminal por parte de quienes se encuentran en la cúspide del aparato de poder, los jerarcas o jefes, no depende pues en absoluto de la "alta disposición al hecho" de los ejecutores directos. El éxito del plan 
Raúl Pariona Arana - El posicionamiento de la teoría de la autoría mediata por organización en la jurisprudencia peruana

criminal radica más bien - como ya lo referí en mis trabajos anteriores - en el dominio que el hombre de atrás ejerce sobre la organización basado en el poder de mando y en el apartamiento de la organización criminal, pues solo así el éxito está asegurado independientemente de la existencia o inexistencia de la disposición al hecho de los ejecutores. Normalmente esta característica acompaña a esta forma de autoría ${ }^{29}$, pero no es un elemento fundamentador, no es pues un presupuesto de esta forma de autoría. Su ausencia, por sí misma, no elimina el dominio del hecho que ejercen los hombres de atrás.

\section{PROPUESTA DE FUNDAMENTACIÓN DE LA AUTORÍA MEDIATA POR ORGANIZACIÓN - CONCEPCIÓN PERSONAL}

La concepción que venimos defendiendo desde hace varios años en diversos trabajos ${ }^{30}$ y que ahora es actualizada y desarrollada, afirma que autor mediato por dominio de organización es quien valiéndose de una organización (aparato de poder organizado) sobre la cual tiene dominio, ordena la comisión de delitos. El dominio sobre la organización le otorga al hombre de atrás un dominio sobre la producción del resultado que materialmente es realizado por un órgano de ejecución del aparato organizado: el ejecutor inmediato. Desde nuestro punto de vista, la autoría mediata en virtud de dominio por organización se fundamenta en el dominio sobre la organización. La estructura organizacional y funcional del aparato debe estar conformada de modo tal que las órdenes de los "jefes supremos", "líderes" o "mandos", sean ejecutadas "automáticamente". La estructura jerárquicamente organizada permite la automaticidad en el cumplimiento de las órdenes. Elementos fundamentales de esta forma de dominio son el poder de mando que tiene el hombre de atrás sobre la organización y el apartamiento del derecho de la organización. Poder de mando y apartamiento del derecho materializan, pues, el dominio que se tiene sobre la organización. Poder de mando sobre y dentro de la organización es el criterio rector; y el apartamiento del derecho es el elemento fundamental que contribuye al dominio sobre la organización, pues solo dentro de organizaciones que están apartadas del derecho, las órdenes ilícitas se cumplen "automáticamente". Ello debido a que una organización que actúa ilegalmente tiene procesos de funcionamiento propios que se distancian de un funcionamiento "normal" y que posibilitan, de esta manera,

\footnotetext{
${ }_{29}$ Visto más detenidamente, este criterio acompaña incluso a gran parte de la autoría en general, es decir, no está limitada a la autoría mediata por organización.

${ }^{30}$ Cfr. mis trabajos publicados en JuS Doctrina \& Práctica, Lima, 12/2007, p. 37 y ss.; en Actualidad Jurídica, Lima, diciembre 2007, t. 169, p. 113 y ss.; y en JuS Jurisprudencia, Lima, 2/2008, p. 145 y ss.; y en: Problemas fundamentales de la parte general del código penal, Lima Friburgo 2009, p. 43 y ss. Una compilación se ofrece en: Autoría mediata por organización, Lima 2009.
} 
Raúl Pariona Arana - El posicionamiento de la teoría de la autoría mediata por organización en la jurisprudencia peruana

el cumplimiento de sus objetivos ${ }^{31}$. Estos elementos son los que posibilitan el dominio del logro del resultado típico, ambos son pues "presupuestos" del dominio por organización y fundamentan por lo tanto la autoría mediata a través de aparatos organizados de poder.

En este tipo de criminalidad por medio de aparatos de poder, la fungibilidad (reemplazabilidad) de los órganos de ejecución de la organización, es decir, la posibilidad de sustituir al ejecutor y la elevada disposición al hecho del ejecutor, son solo características que normalmente acompañan a este tipo de dominio, no lo fundamentan, es decir, su ausencia en el caso concreto no impedirá el dominio del evento criminal por parte de los jefes de los aparatos de poder. La fungibilidad de los ejecutores concurre normalmente en este tipo de criminalidad, pues estos brazos ejecutores constituyen simples piezas en la maquinaria que es la organización. El significado del ejecutor para la organización no es determinante, es simplemente un órgano ejecutor de las "importantes" decisiones de los altos mandos. En esta criminalidad se puede apreciar -no pocas veces- que estos no resultan fácilmente reemplazables en el caso concreto. Sin embargo, este dato no elimina el dominio que ha ejercido el hombre de atrás sobre el evento criminal. Igualmente, se puede observar con frecuencia la disposición al hecho del ejecutor, más aún en organizaciones terroristas donde el factor ideológico juega un papel importante. Sin embargo, si en el caso concreto se constata que no hubo disposición al hecho por parte del ejecutor y pese a ello realiza el evento criminal, este hecho no eliminará el dominio que tuvo el hombre de atrás.

\section{PALABRAS FINALES}

Si se quisiera expresar en pocas palabras el significado de la sentencia de la Corte Suprema en el caso Fujimori debería indicarse que ella consolida la teoría de la autoría mediata por organización en la jurisprudencia nacional e internacional. Esta es la culminación de un desarrollo que empezó hace ya muchos años en la doctrina. Este proceso confirma que la autoría mediata por organización es la estructura dogmática más adecuada para juzgar crímenes cometidos desde y a través de aparatos organizados de poder. 31 Este elemento permitiría a su vez, limitar el ámbito de aplicación de la teoría y de este modo evitar el peligro
de su posible degeneración, garantizando así su legitimidad. 